\title{
ON THE HOMOTOPY PROPERTY OF DEGREE FOR MULTIVALUED NONCOMPACT MAPS
}

\author{
by J. R. L. WEBB
}

(Received 2 March, 1979)

1. Introduction. Over the last few years, various extensions of the topological degree of a mapping have been made so as to include non-compact perturbations of the identity. One such extension, which employs compactness conditions, has been to the class of limit compact maps which were extensively studied by Sadovsky [7]. The class is a large one as it contains all compact mappings, contraction mappings and, more generally, condensing mappings. Sadovsky [7] gives a theory of degree for maps of the form $I-f$, where $f$ is limit compact, and this was extended independently and with different methods by Petryshyn and Fitzpatrick [4] and the author [9] to allow $f$ to be a multi-valued mapping. A refinement of the methods of [9] was given by Vanderbauwhede [8].

A drawback in studying this class is that some of the standard properties of degree fail to hold; for example, that of boundary value dependence. An example where this fails is given by De Pascale and Guzzardi [2]. This means that some of the results of [8] and [9] are in error. This arises because the result that establishes the equality of the degrees of two homotopic limit compact maps on an open set $\Omega$ requires that the whole homotopy be limit compact on the product space $\bar{\Omega} \times[0,1]$. Even in the simplest case when $F$ and $G$ are limit compact, the homotopy $H=t F+(1-t) G$ can fail to be limit compact (as pointed out by Sadovsky [7]).

In the present note we intend to prove a homotopy result which is less stringent. The proof given here is based on the method of [9] but the methods of [4] seem equally applicable. We also prove some other results including an analogue of the classical Rothe fixed point theorem. These results seem to be new.

2. Preliminaries. Throughout $X, Y$ will denote Fréchet spaces; that is, complete, locally convex, topological linear spaces over the reals with topology induced by a translation invariant metric $d$. Let $\Omega$ be a subset of $X$, let $\bar{\Omega}$ denote its closure, $\partial \Omega$ its boundary and $\operatorname{co}(\Omega)$ its convex hull. The $r$-neighbourhood of $\Omega$ is the set

$$
B(\Omega, r)=\{y \in X: \text { there exists } x \in \Omega \text { such that } d(y, x)<r\} .
$$

When $\Omega=\{x\}$ we write $B(x, r)$ for the open ball with centre $x$ and radius $r$. We can, and for simplicity do, assume that $d$ is chosen so that open balls are convex [6; pp. 18-20].

Let $2^{X}$ denote the nonempty subsets of $X$. A multi-valued mapping $F$ from $\Omega$ to $X$ is a function $F: \Omega \rightarrow 2^{X}$ and is identified with its graph. We write $F(x)=\{y \in X:[x, y] \in F\}$, $F(\Omega)=\bigcup_{x \in \Omega} F(X)$. Was say $F$ is upper semicontinuous at $x$ (abbreviated u.s.c.) if $F(x)$ is a closed, convex set and, for each $\varepsilon>0$, there exists $\delta=\delta(x, \varepsilon)>0$ such that $F(B(x, \delta)) \subset$ $B(F(x), \varepsilon) . F$ is u.s.c. on $\Omega$ if this holds for each $x$ in $\Omega$.

Glasgow Math. J. 21, (1980) 125-130. 
We adopt the convention of denoting single-valued mappings by lower case letters and multi-valued ones by capitals.

The limit compact operators are defined in terms of a transfinite sequence of sets $\left\{K_{\alpha}: \alpha\right.$ an ordinal $\}$. Let $D$ be a closed subset of $X$ and let $F: D \rightarrow 2^{X}$ be u.s.c. Let $K_{0}=\overline{c o} F(D)$. Let $\alpha$ be an ordinal and suppose $K_{\beta}$ has been defined for all $\beta<\alpha$. If $\alpha$ is of the first kind let $K_{\alpha}=\overline{c o} F\left(D \cap K_{\alpha-1}\right)$, and if $\alpha$ is of the second kind let $K_{\alpha}=\bigcap_{\beta<\alpha} K_{\beta}$. It is readily shown that $\left\{K_{\alpha}\right\}$ is a decreasing family of sets and so by a theorem of Baire [e.g., [5, p. 132] there exists an ordinal $\gamma$ such that $K_{\alpha}=K_{\gamma}$ for every $\alpha>\gamma$. In particular, $K_{\gamma}=\overline{c o} F\left(D \cap K_{\gamma}\right)$. We say $F$ is limit compact on $D$ if $K_{\gamma}$ is compact or empty. We shall write $K_{F}$ for this limit set.

Sadovsky [7] established a theory of topological degree for maps of the form I-f, where $f$ is limit compact, while Petryshyn and Fitzpatrick [4] and the author [9] independently obtained a similar theory for the multi-valued case by different methods. However, these methods yield the same end product by the author's uniqueness result $[10]$.

We briefly review the definition made in [9] (see also [8]). The degree of $I-F$ with respect to an open set $\Omega$ in $X$ and the point $0, \operatorname{deg}(I-F, \Omega, 0)$ is defined provided $0 \notin(I-F)(\partial \Omega)$.

If $K_{F}=\varnothing$ it is defined to be zero. If $K_{F} \neq \varnothing$, then as $K_{F}=\overline{c o} F\left(\bar{\Omega} \cap K_{F}\right), \bar{\Omega} \cap K_{F}$ is a nonempty, compact subset of $\bar{\Omega}$ and we shall employ the following result of [9]. A similar result, but which seems not to prove enough, is proved in [8], and in [3] it is shown how one can remove the restriction we make on the metric $d$.

Approximation Lemma. Let $A$ be an open subset of $Y$ and let $K$ be a compact subset of $\bar{A}$. Suppose $H: \bar{A} \rightarrow 2^{X}$ is u.s.c. Then, for every $\varepsilon>0$, there exist a neighbourhood $V_{\varepsilon}$ of $K$ and a continuous single-valued map $f_{\varepsilon}: \bar{V}_{\varepsilon} \rightarrow c o F(K)$ with the property that, for each $x \in \bar{V}_{\varepsilon}$ there exists $y \in K$ and $z \in F(y)$ with $d(x, y)<\varepsilon$ and $d\left(f_{\varepsilon}(x), z\right)<\varepsilon$. Moreover, if $K_{1}$ and $K_{2}$ are disjoint compact subsets of $K$, for $\varepsilon$ sufficiently small, there exist neighbourhoods $U_{j}$ of $K_{j}$ such that $f_{\varepsilon}\left(U_{j}\right) \subset \operatorname{co} F\left(K_{j}\right) \cap B\left(F\left(K_{j}\right), \varepsilon\right), j=1,2$.

This result is proved in [9] in the case when $X, Y$ are Banach spaces. However it readily extends to the present case as all that is needed is the convexity of $r$ neighbourhoods, which follows from that of balls (which could be avoided as in [3]), and the existence of a partition of unity subordinate to a finite collection of open sets.

The definition of degree in the case $K_{F} \neq \varnothing$ is now

$$
\lim _{\varepsilon \rightarrow 0} d_{L S}\left(I-f_{\varepsilon}, V_{\varepsilon} \cap \Omega, 0\right),
$$

where $d_{L S}$ denotes the classical Leray-Schauder degree for compact perturbations of the identity and $f_{\varepsilon}, V_{\varepsilon}$ are as above with $K$ taken to be $K_{F} \cap \bar{\Omega}$. That this definition makes sense and is independent of the approximating mappings and neighbourhoods is shown in [9] and [8]. The idea behind this approach is due to Cellina and Lasota [1], who used a similar method for compact multi-valued mappings. 
3. Results. Our new homotopy theorem reads as follows.

THEOREM 1. Let $\Omega$ be an open subset of $X$ and let $H: \bar{\Omega} \times[0,1] \rightarrow 2^{X}$ be u.s.c. Suppose that $F=H(\cdot, 0)$ and $G=H(\cdot, 1)$ are u.s.c. and limit compact on $\bar{\Omega}$ and that there exists a compact convex set $K$ containing $K_{F} \cup K_{G}$ and such that $H((K \cap \bar{\Omega}) \times[0,1]) \subset K$. Then, if $x \notin H(x, t)$ for all $x$ in $\partial \Omega$ and all $t$ in $[0,1]$, then

$$
\operatorname{deg}(I-F, \Omega, 0)=\operatorname{deg}(I-G, \Omega, 0) \text {. }
$$

Proof. If $K \cap \bar{\Omega}=\varnothing$, then both degrees are zero. If $K \cap \bar{\Omega} \neq \varnothing$, then by the approximation theorem, there are neighbourhoods $W_{\varepsilon}$ of $(K \cap \bar{\Omega}) \times[0,1], W_{\varepsilon}^{i}$ of $(K \cap \bar{\Omega}) \times\{j\}$, $(j=0,1)$, and a continuous map $h_{\varepsilon}$ which approximates $H$ on these sets. Then there exists a neighbourhood $U_{\varepsilon}$ of $K \cap \bar{\Omega}$ such that $U_{\varepsilon} \times\{j\} \subset W_{\varepsilon}^{i},(j=0,1)$, and $U_{\varepsilon} \times[0,1] \subset W_{\varepsilon}$; moreover, $f_{\varepsilon}=h_{\varepsilon}(\cdot, 0)$ approximates $F$ and $g_{\varepsilon}=h_{\varepsilon}(\cdot, 1)$ approximates $G$ on $\bar{U}_{\varepsilon}$. We show that, for all $\varepsilon$ sufficiently small, $x \neq h_{\varepsilon}(x, t)$ for all $x$ in $\partial\left(\Omega \cap U_{\varepsilon}\right)$ and $t$ in $[0,1]$. Indeed, $x=h_{\varepsilon}(x, t)$ implies that $x$ is in $K$ as $h_{\varepsilon}\left(W_{\varepsilon}\right) \subset K$. Therefore $x \neq h_{\varepsilon}(x, t)$ for all $x \in \partial U_{\varepsilon}$. Also, if there were sequences $\varepsilon_{j} \rightarrow 0,\left\{t_{j}\right\} \subset[0,1],\left\{x_{j}\right\} \subset \partial \Omega$ such that $x_{j}=h_{\varepsilon_{j}}\left(x_{j}, t_{j}\right)$, by compactness of $K$ we can suppose $x_{j} \rightarrow x \in \partial \Omega, t_{j} \rightarrow t$ and so $h_{\varepsilon_{j}}\left(x_{i}, t_{j}\right) \rightarrow x$. By the u.s.c. of $H$ this implies $x \in H(x, t)$, a contradiction (see e.g. Lemma 1 of [9]). By the homotopy property of Leray-Schauder degree this proves

$$
d_{L S}\left(I-f_{\varepsilon}, U_{\varepsilon} \cap \Omega, 0\right)=d_{L S}\left(I-g_{\varepsilon}, U_{\varepsilon} \cap \Omega, 0\right) .
$$

Using the facts that $f$ approximates $F$ and $x \in F(x)$ implies that $x \in K_{F}$, the excision property of $d_{L S}$ now shows that we can replace $U_{\varepsilon}$ by any neighbourhood of $K_{F}$ in the term on the left so this term equals $\operatorname{deg}(I-F, \Omega, 0)$. Likewise the term on the right is the degree of $I-G$.

Remarks. (1) $K=K_{H}(\bar{\Omega} \times[0,1])$ always satisfies the invariance property needed in the theorem. When $K_{H}$ is compact we obtain the known homotopy result. The point of our result is that $K$ may be smaller than $K_{H}$ so more possible homotopies are admitted. See the following corollaries and example.

(2) The methods of [4] seem to work equally well and using results from [4] one can give an equally simple proof.

Corollary 1. Suppose $F, G$ are limit compact and u.s.c. and that there exists a compact convex set $K$ which contains $K_{G}$ for which $F(K \cap \bar{\Omega}) \subset K$ and $G(K \cap \bar{\Omega}) \subset K$. Then if $x \notin t F(x)+(1-t) G(x)$ for all $x \in \partial \Omega$, all $t \in[0,1]$, and $\operatorname{deg}(I-G, \Omega, 0)$ is nonzero, there exists a fixed point of $F$; that is, $x \in \Omega$ such that $x \in F(x)$.

Proof. Let $H(x, t)=t F(x)+(1-t) G(x) ; H$ is u.s.c. and $H(K \cap \bar{\Omega} \times[0,1]) \subset K$. By the proof of Theorem 1 , there are neighbourhoods $U_{\varepsilon}$ of $K \cap \bar{\Omega}$ and mappings $f_{\varepsilon}=h_{\varepsilon}(\cdot, 0)$ and $g_{\varepsilon}=h_{\varepsilon}(\cdot, 1)$ which approximate $F$ and $G$ on $\bar{U}_{\varepsilon}$. Moreover,

$$
\begin{aligned}
d_{L S}\left(I-f_{\varepsilon}, U_{\varepsilon} \cap \Omega, 0\right) & =d_{L S}\left(I-g_{\varepsilon}, U_{\varepsilon} \cap \Omega, 0\right) \\
& =\operatorname{deg}(I-G, \Omega, 0) \neq 0
\end{aligned}
$$


for $\varepsilon$ sufficiently small. Therefore there exist $x_{\varepsilon} \in U_{\varepsilon}$ such that $x_{\varepsilon}=f_{\varepsilon}\left(x_{\varepsilon}\right) \subset K$. It follows that $x_{\varepsilon} \rightarrow x$ and $x \in F(x)$.

Remark. Corollary 1 extends a similar result of De Pascale-Guzzardi [2] obtained for condensing maps. We cannot assert $\operatorname{deg}(I-F, \Omega, 0) \neq 0$ as we made no assumption on $K_{F}$. It follows from this proof that $K_{\mathrm{F}} \neq \varnothing$. We could apply such a result if $G$ were odd on $\bar{\Omega}$, that is $G(-x)=-G(x)$, for then $\operatorname{deg}(I-G, \Omega, 0)$ is odd if it is defined [4].

COROLlary 2. If $F, G$ are u.s.c. and limit compact on $\bar{\Omega}$ and if $K_{F} \subset K_{G}, F\left(K_{G} \cap \bar{\Omega}\right) \subset$ $K_{G}$, and $x \notin t F(x)+(1-t) G(x)$ for all $x \in \partial \Omega$ and $t \in[0,1]$, then

$$
\operatorname{deg}(I-F, \Omega, 0)=\operatorname{deg}(I-G, \Omega, 0) .
$$

Proof. Let $K=K_{G}$ and $H(x, t)=t F(x)+(1-t) G(x)$ and apply the theorem.

Corollary 3. Suppose $F, G$ are as in Corollary 2 but with the requirement $x \in \partial \Omega$ and $(x-F(x)) \cap \lambda(x-G(x)) \neq \varnothing$ imply $\lambda>0$. Then $\operatorname{deg}(I-F, \Omega, 0)=\operatorname{deg}(I-G, \Omega, 0)$, ifboth degrees are defined.

Proof. It is readily verified that $x \notin t F(x)+(1-t) G(x)$ for $x \in \partial \Omega$ and $0 \leq t \leq 1$.

Remark. Corollary 2 applies in the special case $F(x) \subset G(x)$ for all $x$ in $\bar{\Omega}$. This gives a result of Vanderbauwhede [8]. It also applies in the special case $K_{F}=K_{G}$.

The following example shows how our theorem applies but the homotopy $H$ is not limit compact.

EXAMPLE. Let $X=\left(c_{0}\right)$ the space of all sequences of real numbers that converge to zero with the norm $\|x\|=\sup \left|x_{n}\right|$ for $x=\left(x_{n}\right)$. Let $\Omega$ be the open unit ball of $X$ and for $x=\left(x_{1}, x_{2}, \ldots\right), f(x)=\left(\|x\|, x_{1}, x_{2}, \ldots\right)$ and $g(x)=\left(0, x_{1}, x_{2}, \ldots\right)$. Then an easy calculation shows that $K_{\mathrm{f}}=K_{\mathrm{g}}=\{0\}$, and, if we set $h(x, t)=t f(x)+(1-t) g(x), x \neq h(x, t)$ for all $x \in \partial \Omega$ and all $t \in[0,1]$. Moreover,

$$
K_{h}=\left\{\left(s_{1}, s_{2}, \ldots,\right), \text { where } 0 \leq s_{j} \leq 1 \text { and } s_{j} \rightarrow 0 \text { as } j \rightarrow \infty\right\},
$$

a noncompact set. Indeed, we have

$$
\begin{aligned}
K_{0} & =\overline{c o h}(\bar{\Omega} \times[0,1]) \\
& =\overline{c o}\left\{\left(t\|x\|, x_{1}, x_{2}, \ldots\right), 0 \leq t \leq 1,-1 \leq x_{i} \leq 1\right\} \\
& =\left\{\left(s, x_{1}, x_{2}, \ldots\right), 0 \leq s \leq 1,-1 \leq x_{j} \leq 1\right\} .
\end{aligned}
$$

To see this certainly $\left(0, x_{1}, x_{2}, \ldots\right)$ is in $K_{0}$. As $\left(1, x_{2}, x_{3}, \ldots\right)$ and $\left(-1, x_{2}, x_{3}, \ldots\right)$ lie in $\bar{\Omega}$, we have $\left(1,1, x_{2}, x_{3}, \ldots\right)$ and $\left(1,-1, x_{2}, x_{3}, \ldots\right)$ are in $K_{0}$. By convexity, $\left(1, x_{1}, x_{2}, x_{3}, \ldots\right) \in K_{0}$ and this proves the assertion about $K_{0}$. That $K_{h}$ is the set asserted follows in an obvious manner from this.

REMARKs. (1) In this example one can obtain the same result by proving that $f$ and $g$ are both homotopic to the zero map. These homotopies are limit compact. Even so, it is considerably easier to apply our theorem. In fact, Theorem 3 that follows gives the result immediately. 
(2) Our result is really for those limit compact maps which are not in some better behaved subclass such as $\Phi$-condensing (see. e.g. [4]), or compact, for in these cases more can usually be said. If one has to calculate the limit set, then our theorem usually requires no further work whereas it could be rather difficult to calculate $K_{H}$.

We now obtain an analogue of Rothe's theorem which extends some results of Petryshyn and Fitzpatrick [4].

THEOREM 2. Let $\Omega$ be an open convex set and let $G$ be u.s.c. and limit compact on $\bar{\Omega}$. Suppose $K_{G} \neq \emptyset$ and that $G\left(\partial \Omega \cap K_{G}\right) \subset \bar{\Omega}$ (no restriction if $K_{G} \subset \Omega$ ). Then $G$ has a fixed point in $\vec{\Omega}$.

Proof. If there exists a point $x_{0} \in K_{G} \cap \Omega$ we let $H(x, t)=t G(x)+(1-t) x_{0}$. We can suppose $x \notin G(x)$ for all $x \in \partial \Omega$. Then $x \in H(x, t)$ implies that $x \in K_{G}$ (proof by transfinite induction) so $x \in H(x, t), x \in \partial \Omega$ would imply $x=t u+(1-t) x_{0}, u \in G\left(\partial \Omega \cap K_{G}\right) \subset \bar{\Omega}$. As $x_{0} \in \Omega$ and $\Omega$ is convex this is impossible for $t<1$ and $t=1$ is excluded above. By Corollary $2, \operatorname{deg}(I-G, \Omega, 0)=\operatorname{deg}\left(I-x_{0}, \Omega, 0\right)$. By uniqueness of topological degree the right hand side coincides with the Leray-Schauder degree and equals 1 . This proves that there exists $x \in G(x), x \in \Omega$ in this case. If no such $x_{0}$ exists then $K_{G} \cap \partial \Omega=K_{G} \cap \bar{\Omega}$ is a nonempty compact convex set and is invariant under $G$. By the analogue of the Schauder fixed point theorem for compact maps [1], $G$ has a fixed point.

Remark. The restriction that $K_{G}$ be nonempty is a necessary one. For example, consider $X=\left(c_{0}\right)$ and $g(x)=\left(1, x_{1}, x_{2}, \ldots\right)$. Then $g$ maps the unit ball into itself but has no fixed point. Also, the example $f(x)=\left(3-3 x_{1}, 0, x_{2}, x_{3}, \ldots\right)$ in $\left(c_{0}\right)$ with $K_{f}=$ $\{(t, 0,0, \ldots), 0 \leq t \leq 3\}$ shows that the hypothesis $f\left(\partial \Omega \cap K_{f}\right) \subset \bar{\Omega}$ is less stringent than $f(\partial \Omega) \subset \bar{\Omega}$.

Employing a similar idea to that of Theorem 2 gives the following result.

Theorem 3. Let $\Omega$ be open and let $F, G$ be u.s.c. and limit compact on $\bar{\Omega}$. Suppose $K_{F}$ and $K_{G}$ are nonempty subsets of $\Omega$, Then

$$
\operatorname{deg}(I-F, \Omega, 0)=\operatorname{deg}(I-G, \Omega, 0)=1 .
$$

Proof. Let $x_{0} \in K_{F}$ and let $H(x, t)=t F(x)+(1-t) x_{0}$. As $x \in H(x, t)$ implies $x \in K_{F} \subset \Omega$ we have $x \notin H(x, t)$ for all $x \in \partial \Omega$. By Corollary $2, \operatorname{deg}(I-F, \Omega, 0)=\operatorname{deg}\left(I-x_{0}, \Omega, 0\right)=1$. Similarly we prove the result for $G$.

Remark. Theorem 3 does not need Theorem 1 for its proof; $H$ is a limit compact homotopy in this case.

\section{REFERENCES}

1. A. Cellina and A. Lasota, A new approach to the definition of topological degree for multi-valued mappings, Atti. Accad. Naz. Lincei. Rend. Cl. Sci. Fis. Mat. Natur. 47 (1969), 434-440.

2. E. De Pascale and R. Guzzardi, On the boundary values dependence for the topological degree of multi-valued noncompact maps, Boll. Un. Mat. Ital. 13-A (1976), 110-116. 
3. P. M. Fitzpatrick and W. V. Petryshyn, Fixed point theorems and the fixed point index for multivalued mappings in cones, J. London Math. Soc. (2) 12 (1975), 75-85.

4. P. M. Fitzpatrick and W. V. Petryshyn, A degree theory, fixed point theorems, and mapping theorems for multi-valued noncompact mappings, Trans. Amer. Math. Soc. 194 (1974), $1-25$.

5. E. Kamke, Theory of sets (Dover, 1950).

6. W. Rudin, Functional analysis (McGraw-Hill, 1973).

7. B. N. Sadovsky, Limit compact and condensing operators (English translation), Russian Math. Surveys, 27, (1972), 85-155.

8. A. Vanderbauwhede, On a modified degree theory for multi-valued mappings, Simon Stevin, 50 (1976-77), 65-86.

9. J. R. L. Webb, On degree theory for multi-valued mappings and applications, Boll. Un. Mat. Ital. 9 (1974), 137-158.

10. J. R. L. Webb, On uniqueness of topological degree for set-valued mappings, Proc. Roy. Soc. Edinburgh, Sect. A 74 (1974-75), 225-229.

DePARTMENT OF Mathematics

INDIANA UNIVERSITY

BLOOMINGTON

INDIANA 47405

Present Address:

Department of Mathematics

UNIVERSITY OF GLASGOW 